\title{
INFINITE SERIES AND THE DERIVED SET \\ OF THE AGGREGATE OF THE FRACTIONAL PARTS \\ OF ITS PARTIAL SUMS: ADDENDUM
}

\author{
S. Audinarayana Moorthy
}

In the author's paper [1] it was proved that the fractional parts of the partial sums of an infinite series (of real terms) diverging to $+\infty$ or $\ldots \infty$, in which the general term tends to zero, are everywhere dense in the closed unit interval. This result was extended to series of infinite oscillation (see Remark 4.1 of the said paper) on the argument that a sequence of partial sums having infinite oscillation has a subsequence that diverges to $+\infty$ or $-\infty$. However, it remains to be shown that this subsequence is the sequence of partial sums of some infinite series diverging to $+\infty$ or $-\infty$, in which the $n$th term tends to zero; and the purpose of this addendum is to prove this result in the form of the following :

THEOREM. Let $\left(S_{n}\right)$ be a sequence of real terms such that $s_{n}-s_{n-1} \rightarrow 0$. If $\left(s_{n}\right)$ oscillates between finite and infinite limits or. between $+\infty$ and $-\infty$, then $\left(s_{n}\right)$ has a subsequence $\left(S_{m_{i}}\right)_{i=1}^{\infty}$ which diverges to $+\infty$ or $-\infty$ and is such that $S_{m_{i}}-S_{m_{i-1}} \rightarrow 0$.

Proof. We prove the theorem when the sequence $\left(s_{n}\right)$ oscillates between a positive number $a$ and $+\infty$. The proofs in other cases are similar.

We know (see [2], Part I, Chapter 3, Problem 100, p. 23) that for an

Received 4 December 1981. 
oscillatory series in which the $n$th term tends to zero the sequence of partial sums is everywhere dense between its limit superior and limit inferior. Thus the terms of $\left(S_{n}\right)$ are everywhere dense between $a$ and $\infty$.

Consider the sequence $\left(T_{n}\right)$ where

$$
T_{n}=a+(a / 2)+(a / 3)+\ldots+(a / n) .
$$

This sequence diverges to $\infty$ and is such that $T_{n}-T_{n-1} \rightarrow 0$. Let $\left(\Delta_{n}\right)$ be a sequence of non overzapping open intervals such that $T_{n} \in \Delta_{n}$ for every $n$. Since $a \leq T_{n}$ for each $n$, every member of $\left(T_{n}\right)$ can be realised as the limit of a subsequence of $\left(S_{n}\right)$. Now, since each neighbourhood $\Delta_{n}$ of $T_{n}$ contains infinitely many members of $\left(S_{n}\right)$, we can construct a subsequence $\left(S_{m_{i}}\right)_{i=1}^{\infty}$ of $\left(S_{n}\right)$ such that $S_{m_{i}} \epsilon \Delta_{i}$ for each $i$. Clearly the subsequence $\left(S_{m_{i}}\right)$ is an increasing one and diverges to $\infty$. Also,

$$
0<S_{m_{i}}-S_{m_{i-1}}<T_{i+1}-T_{i-2}
$$

so that $S_{m_{i}}-S_{m_{i-1}} \rightarrow 0$.

The proof of the theorem is now complete.

\section{References}

[1] S. Audinarayana Moorthy, "Infinite series and the derived set of the aggregate of the fractional parts of its partial sums", Buzz. Austral. Math. Soc. 21 (1980), 253-264.

[2] G. Pólya, G. Szegö, Problems and theorems in analysis. Volume I: Series, integral calculus, theory of functions (translated by D. Aeppli. Die Grundlehren der mathematischen Wissenschaften, 193. Springer-Verlag, Berlin, Heidelberg, New York, 1972). Department of Mathematics, Kendrapara College, Kendrapara 754211 , Cuttack Dt., Orissa, India. 